\title{
System Dynamics Study on Knowledge Interaction Behavior in the Interdisciplinary Team

\author{
Xue $\mathrm{CHENG}^{\mathrm{a}}$, Qing-Pu ZHANG ${ }^{\mathrm{b},{ }^{*}}$
}

\author{
School of management, Harbin Institute of Technology, Harbin, 150080, P. R. China \\ achengxue0419@126.com, bzqp2015@hotmail.com,
}

${ }^{*}$ Corresponding author

Keywords: Interdisciplinary team, Knowledge interaction, Innovation ability, System dynamics.

\begin{abstract}
Based on the analysis of influencing factors of knowledge interaction, a system dynamics model of knowledge interaction in the interdisciplinary team is constructed. And Vensim PLE is used to simulate and test the sensitivity of the model. The results show that as the depth of knowledge interaction increases, the team innovation ability gradually increases. With the interaction frequency increasing, the team's knowledge accumulation and innovation ability will gradually increase. However, when the interaction frequency reaches a certain level, the knowledge accumulation of the team will not increase and the team's innovation ability will decline. This model could validly reflect the process of knowledge interaction in the interdisciplinary team, and provide effective decision support for similar knowledge interaction process.
\end{abstract}

\section{Introduction}

Knowledge interaction is an important way for interdisciplinary teams to accomplish their tasks, solve problems and innovate [1]. Many research universities have established interdisciplinary teams. However, not all teams can achieve the desired results and complete the research projects successfully. Due to the influence of management system and team members' own concepts, the cooperation effect of some interdisciplinary teams is not satisfactory. "Free riding", false acceptance, passive coping and other phenomena often appear in the team, which are contrary to the original intention of interdisciplinary team's building. Some scholars believe that the reason of task fail in many interdisciplinary teams is not for lack of some professional knowledge, but because there are some miscommunications on key issues [2,3]. Therefore, the members of interdisciplinary team must consider how to effectively interact to solve problems and improve the team's own innovation ability.

Some scholars have studied the role of knowledge sharing, knowledge transfer, knowledge integration to complete the task and improve the ability of team innovation. For example, Grant [4] emphasizes the role of knowledge integration in organizational innovation. Toedtling et al. [5] studied the impact of knowledge interaction on innovation ability. Pontis and Blandford [6] underline the iterative and interactive information seeking in the process of knowledge construction. Some studies also analyzed the impact of team knowledge interaction. For example, based on grounded theory, Wang et al. [7] demonstrated that environment, sharing channels, knowledge resource requirements and knowledge resource pools have a certain impact on the knowledge interaction among organizations. Li [8] studied the impact of team introspection on the team's ability to interact with each other based on the interactive memory system. Sun [9] studied the impact of team heterogeneity on knowledge sharing. Based on these studies, this paper will use system dynamics method to simulate the knowledge interaction behavior in the interdisciplinary teams, and explore the interaction among variables in order to have a more comprehensive understanding of the knowledge interaction.

\section{Analysis of the Influencing Factors of Knowledge Interaction}

Knowledge interaction in the interdisciplinary team is a complex process, which is influenced by such factors as the depth of knowledge interaction, knowledge absorptive capacity, the degree of 
knowledge matching, the trust among members, interaction frequency and so on. Sometimes, though the team carries on a lot of knowledge interaction, it is just a mere formality and does not explore the essence of the problem, which results in unsatisfactory interaction. In the process of interaction, if members can think independently and critically learn each other's knowledge and integrate into their own knowledge structure to make decisions and solve problems, the effect of knowledge interaction will be greatly improved. Knowledge absorptive capacity has four dimensions, including acquisition, digestion, transformation and utilization [10]. Any weakening of capacity will have a bad impact on the knowledge interaction. Trust has a significant role in promoting knowledge interaction. The higher the degree of trust among members, the more conducive to the communication and exchange among members, and interaction is more extensive and deeper. In addition, the degree of knowledge matching also has an important impact on knowledge interaction. Due to the different knowledge backgrounds among members, there are differences between members' knowledge structure and knowledge stock. If the span of professional knowledge is too large, there is knowledge sticking when different professional members communicate. Besides, the frequency of knowledge interaction should be within a reasonable range. If interaction frequency is low, knowledge among members cannot be effectively transformed. Especially some tacit knowledge cannot be absorbed by others. If interaction frequency is too high, some of the ideas and concepts among members will gradually converge, which is not innovative and lost the meaning of interaction. In both cases, knowledge interaction will not work well.

\section{The System Dynamics Model of Knowledge Interaction}

\section{Basic Assumptions}

The dynamic structure is formed among the influencing factors of knowledge interaction and evolves in the system according to certain rules. To ensure the rationality and feasibility of the research, the following basic assumptions are proposed.

a. Knowledge interaction in the interdisciplinary teams is constantly changing as the team operates.

b. Knowledge interaction involves the process of knowledge transfer, knowledge sharing and knowledge integration. To simplify the model, we treat them as a whole variable.

c. When team interaction frequency is more than 0.5 times per day, knowledge interaction accumulation will not increase.

d. Teams can continually obtain extrinsic knowledge.

\section{Cause and Effect Analysis of Knowledge Interaction}

Based on the above analysis, a cause-effect diagram of knowledge interaction is constructed, and mainly includes the following two feedbacks, as shown in Fig. 1.

Team innovation ability $\rightarrow$ team performance $\rightarrow$ team satisfaction $\rightarrow$ trust coefficient $\rightarrow$ trust $\rightarrow$ communication $\rightarrow$ knowledge interaction $\rightarrow$ knowledge accumulation $\rightarrow$ the total amount of team knowledge $\rightarrow$ the improvement of innovation ability $\rightarrow$ team innovation ability

Team innovation ability $\rightarrow$ competitive pressure $\rightarrow$ knowledge acquisition desire $\rightarrow$ external knowledge acquisition $\rightarrow$ the total amount of team knowledge $\rightarrow$ the improvement of innovation ability $\rightarrow$ team innovation ability

The first feedback is positive feedback. The more the knowledge interaction accumulation, the more conducive to the improvement of team innovation ability. The improvement of innovation ability will increase the satisfaction and trust among team members. It will promote the communication and interaction among members and increase the accumulation of interactive knowledge. The second feedback is negative feedback. The improvement of innovation ability will reduce the team's competitive pressures, which in turn makes the team's external knowledge acquisition and the total amount of team knowledge reduce, and weakens team innovation ability. 


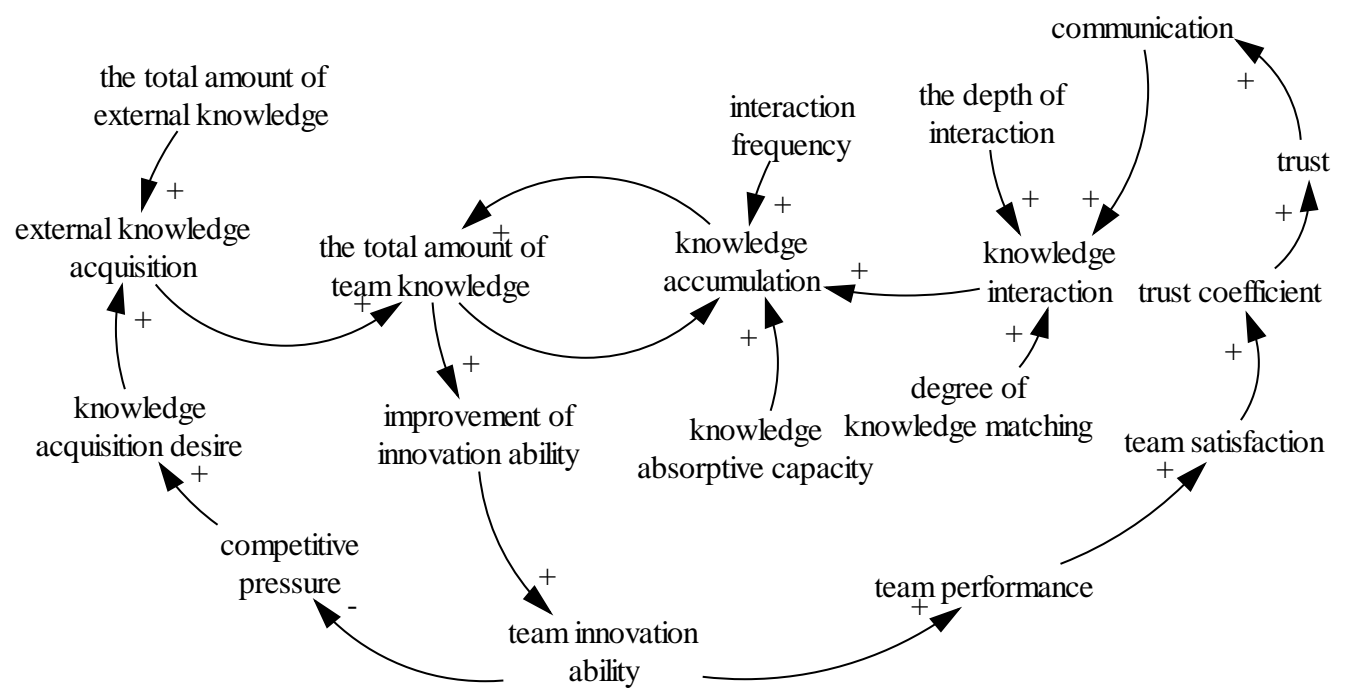

Fig.1 Causal analysis of knowledge interaction

\section{System Flow Chart of Knowledge Interaction}

Based on the feasibility of computer simulation, a system flow chart of knowledge interaction in the interdisciplinary team is obtained by optimizing the cause-effect diagram, as shown in Fig. 2. The system flow chart contains 2 state variables, 5 flow rate variables, 8 auxiliary variables and 10 constants.

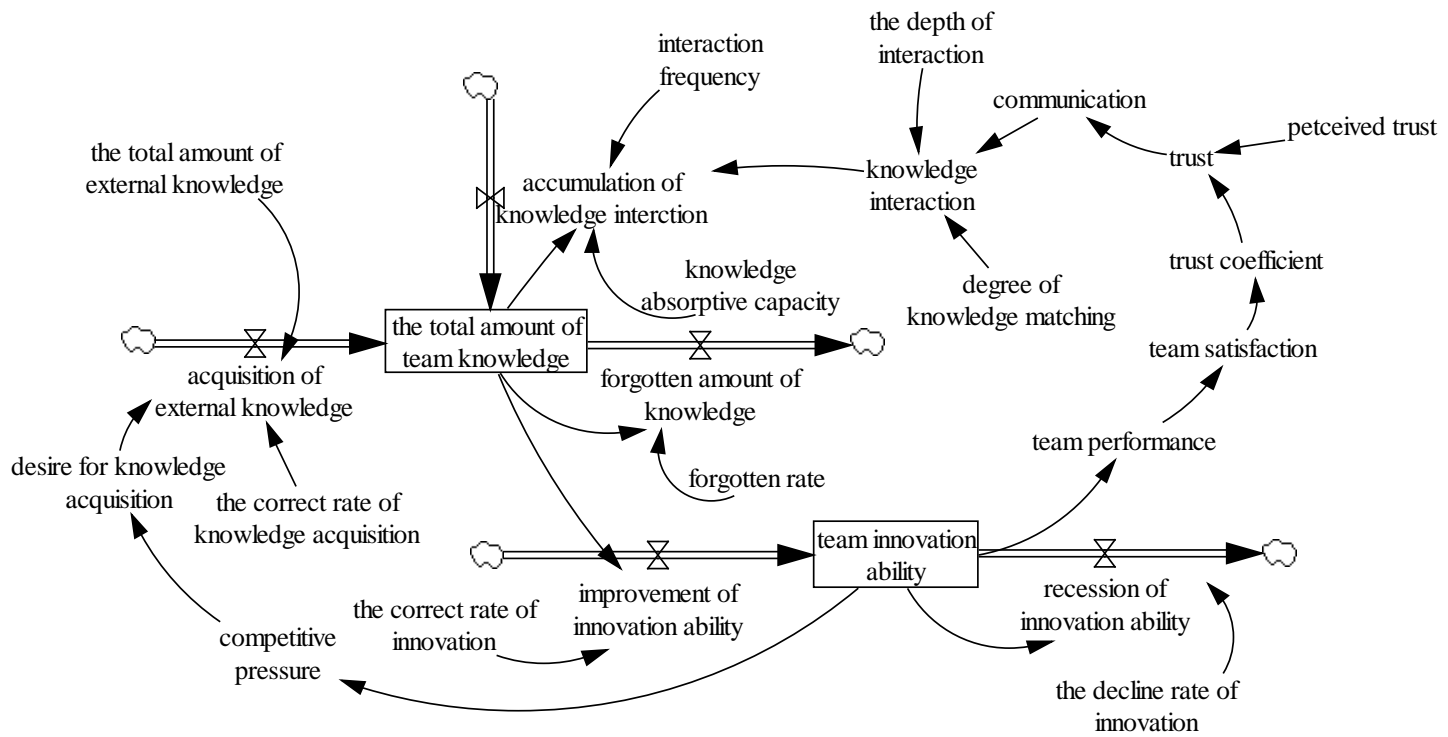

Fig.2 System flow chart of knowledge interaction

\section{The Main Equations and Design Descriptions}

The total amount of team knowledge=INTEG(Acquisition of external knowledge + Accumulation of knowledge interaction - Forgotten amount of knowledge, 20)

Forgotten amount of knowledge=STEP(The total amount of team knowledge $\times$ Forgotten rate, 10)

Forgotten rate $=0.03$

Acquisition of external knowledge $=$ The total amount of external knowledge $\times$ Desire for knowledge acquisition $\times$ The correct rate of knowledge acquisition

The total amount of external knowledge $=100$

The correct rate of knowledge acquisition $=0.3$ 
Accumulation of knowledge interaction=DELAY1I(IF THEN ELSE(Interaction frequency $<=0.5$, The total amount of team knowledge $\times$ Knowledge interaction $\times$ Knowledge absorptive capacity $\times$ Interaction frequency,0),2,0)

When the interaction frequency is greater than 0.5 , the accumulation of knowledge interaction no longer increases. In the actual process of team interaction, if the interaction frequency is too much, team members do not have independent thinking time, resulting in bad interaction effect.

Interaction frequency $=0.3$

Knowledge absorptive capacity $=0.5$

Knowledge interaction $=$ The depth of interaction $\times$ communication $\times$ Degree of knowledge matching

The depth of interaction $=0.6$

Degree of knowledge matching $=0.5$

Communication=WITH LOOKUP (Trust, $([(0,0)-(1,1)],(0,0),(1,0.7)))$

Trust $=$ Perceived trust $\times$ Trust coefficient

Perceived trust $=0.2$

Team innovation =INTEG(The improvement of innovation- The recession of innovative, 10)

The improvement of innovation $=$ The total amount of team knowledge $\times$ The correct rate of innovation

The correct rate of innovation $=0.2$

The recession of innovative $=\mathrm{STEP}($ Team innovation $\times$ The decline rate of innovation, 5 )

The decline rate of innovation $=0.1$

Team performance=WITH LOOKUP (Team innovation ([(0,0)-(2500,10)], $(0,0),(500,2)$, $(1000,5),(2500,8)))$

Team satisfaction=WITH LOOKUP (Team performance, ([(0,0)-(50,1)], $(0,0.2),(50,0.8)))$

Trust coefficient=WITH LOOKUP (Team satisfaction, ([(0,0)-(1,0.9)], $(0,0),(0.3,0.2),(0.5,0.4)$, $(0.6,0.55),(0.8,0.7),(1,0.8)))$

Competitive pressure $=$ WITH LOOKUP(Team innovation, $([(0,0)-(2500,1)],(0,1),(1000,0.8)$, $(2500,0.5)))$

Desire for knowledge acquisition =WITH LOOKUP(Competitive pressure, $([(0,0)-(1,1)],(0,0.1)$, $(0.3,0.5),(1,0.9)))$

\section{Model Simulation and Sensitivity Analysis}

\section{Simulation Results of the Model under the Given Parameters}

As can be seen from Fig. 3, the model can better simulate the dynamic process of knowledge interaction. The total amount of team knowledge, accumulation of knowledge interaction and the team innovation ability remain stable after increasing to a certain value with the advance of simulation results. External knowledge acquisition also shows a gentle trend after gradually declining. This is because in the early stage of the project, the team's interaction level is low. With the development of the project, the ability of interaction and knowledge absorption among teams' members has been continuously improved, and accumulation of knowledge interaction and the total amount of team knowledge continues to increase. As a result, team innovation ability has been greatly improved. Due to the limited knowledge level of team members, when the accumulation of knowledge interaction reaches a certain level, it will not increase any more, which makes the total amount of team knowledge and team innovation ability tend to be stable. 


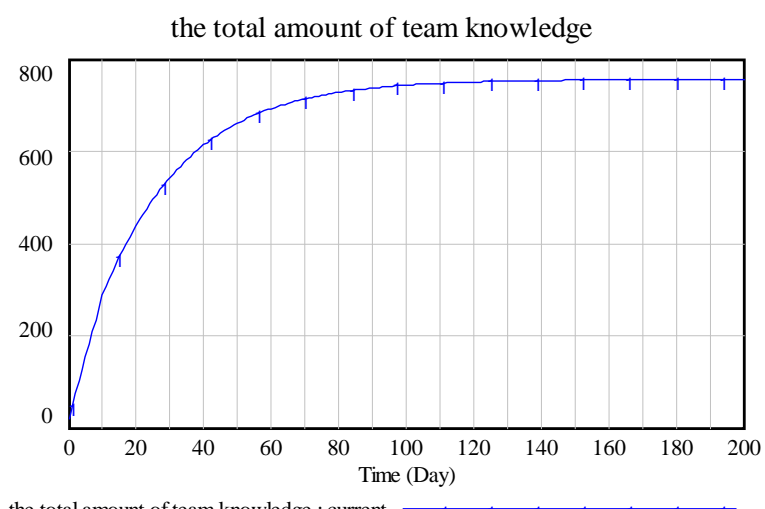

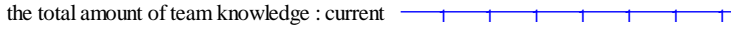

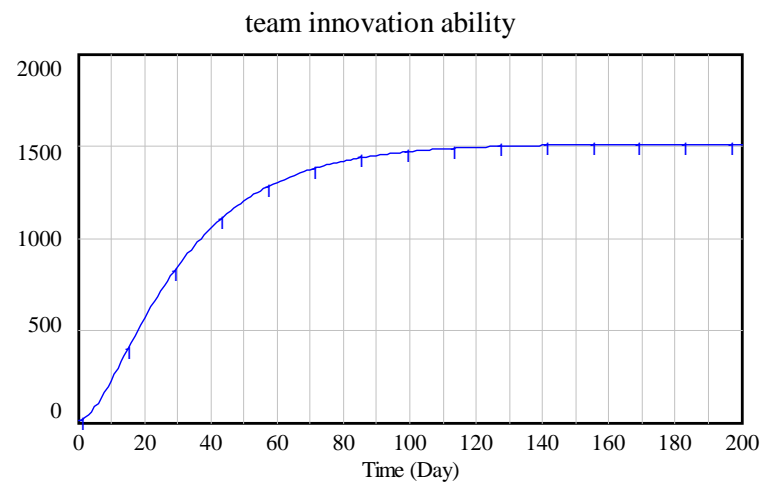

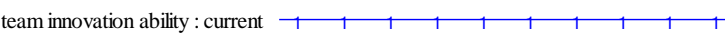

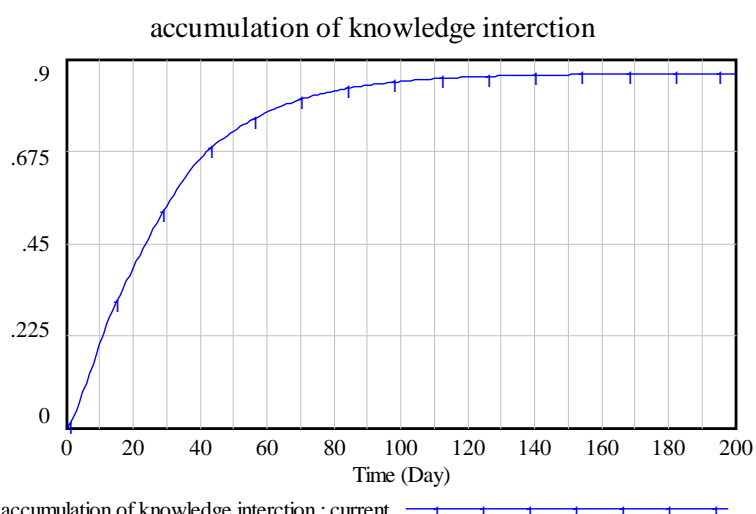

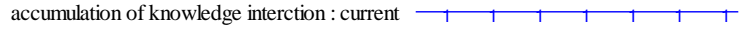

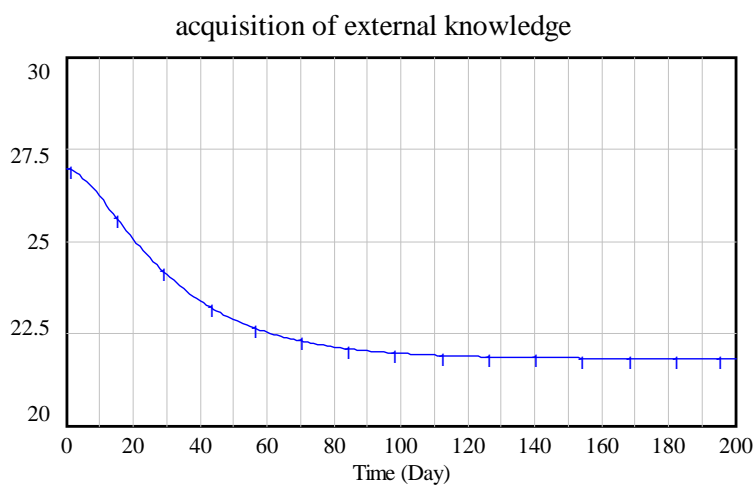

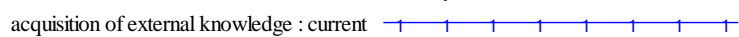

Fig.3 Simulation results of the model under the given parameters

\section{Sensitivity Analysis}

\section{Change the Depth of Knowledge Interaction}

Keep the other parameters unchanged, and adjust the parameter values of the interaction depth from 0.6 to 0.8 and 0.3 , respectively, recorded as test 1 and test 2 . As can be seen from Fig. 4, compared test 1 with the original program and test2, there is a significant gap in the accumulation of knowledge interaction and team innovation ability. This shows that the depth of knowledge interaction has a positive impact on knowledge interaction, thus the team innovation ability increases. Based on this, in the actual interdisciplinary team, the team can increase the depth of knowledge interaction at the beginning of the project and significantly improve the team's innovation ability. When the depth of knowledge interaction researches to a certain extent, team would better not waste too much time and energy to pursuit the small improvement of team innovation ability.

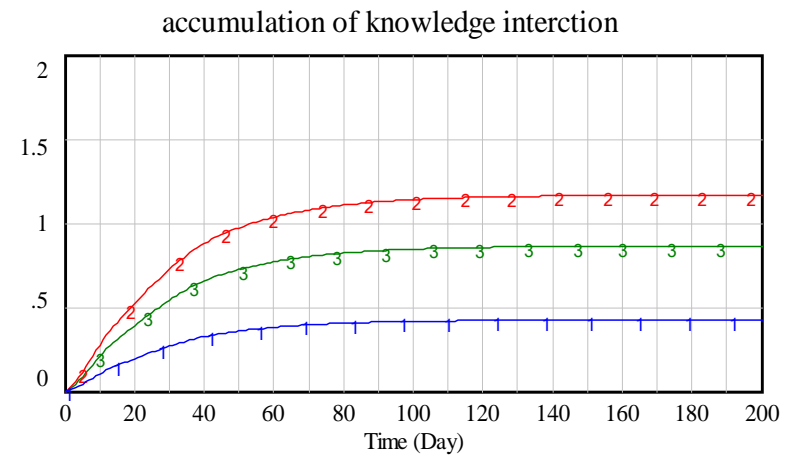

accumulation of knowledge interction : test2

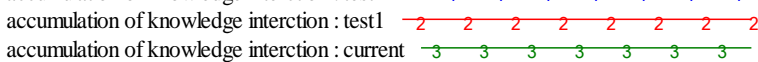

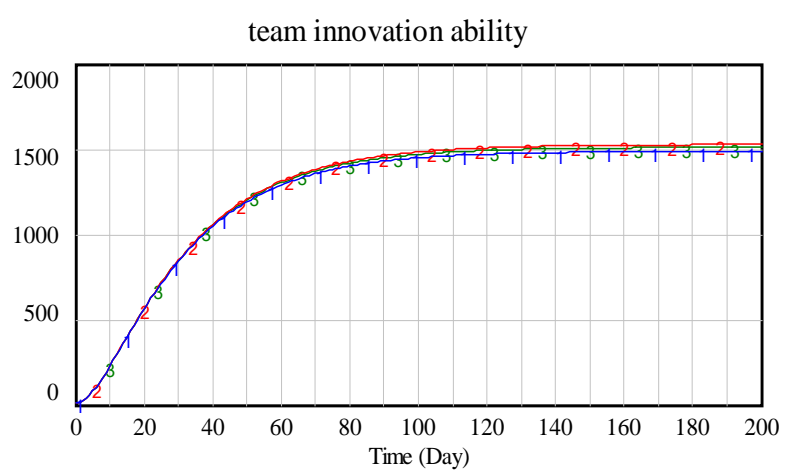

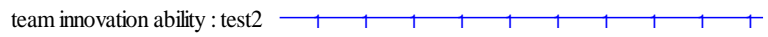
team innovation ability : test1 2 team innovation ability : current

Fig.4 Sensitivity analysis of knowledge interaction depth 


\section{Chang Knowledge Interaction Frequency}

Keep the other parameters unchanged, and adjust the parameter values of knowledge interaction frequency from 0.3 to 0.5 and 0.6 , respectively, recorded as test 1 and test2. From Fig. 5 we can see that, the accumulation of knowledge interaction has significantly increased compared test1 with the original program. The interaction frequency of test 2 is greater than 0.5 , the accumulation of knowledge interaction does not increase. Test1 has a slight increase over test 2 and the original program in the team innovation ability. This shows that in the actual interdisciplinary team, team should find the maximum frequency of knowledge interaction. When the actual interaction frequency is less than the set limit, the team's knowledge accumulation and team innovation ability will gradually increase with the increasing of interaction frequency. When the actual interaction frequency is greater than the set limit, the team's knowledge accumulation will not increase, and team's innovation ability will decline.
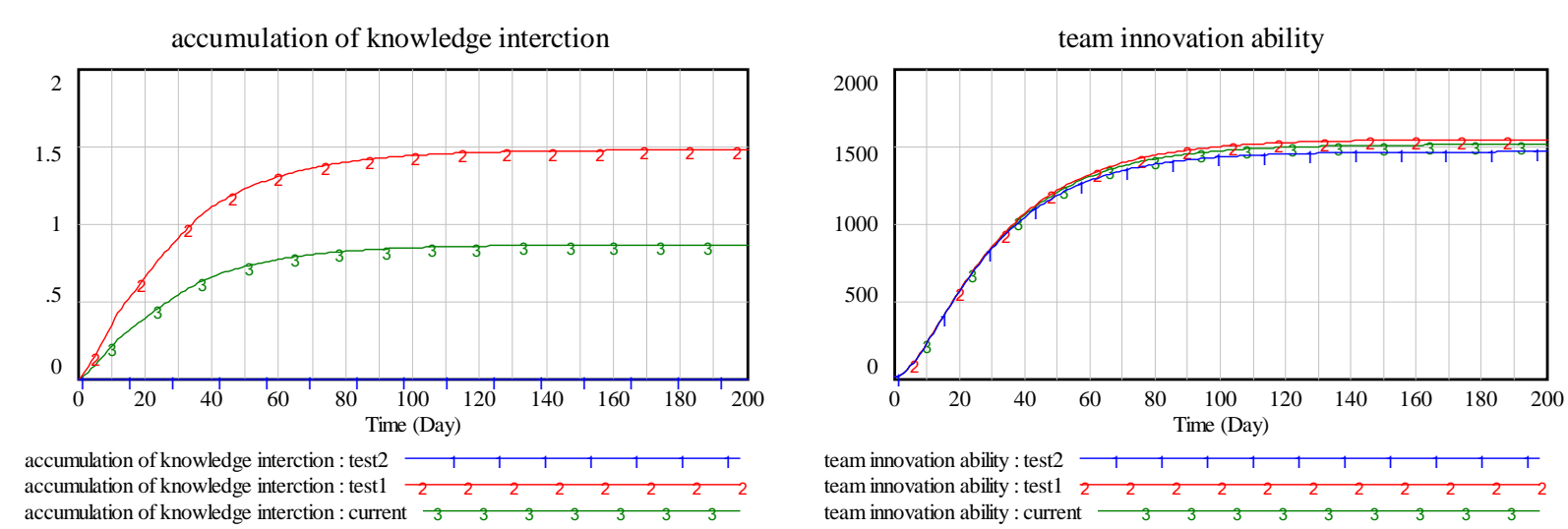

team innovation ability : current

Fig.5 Sensitivity analysis of knowledge interaction frequency

\section{Conclusion}

Based on the analysis of the influencing factors of knowledge interaction, this paper constructs a system dynamics model of knowledge interaction in the interdisciplinary team. Vensim PLE software is used to realize the system simulation and sensitivity analysis of the two parameters, and some policy recommendations are proposed.

From the simulation results, with the increase of the depth of knowledge interaction, the accumulation of knowledge interaction and team innovation ability gradually increases; as the interaction frequency increases, the team's knowledge accumulation and team innovation ability also gradually increase; but when the interaction frequency reaches a certain level, the accumulation of knowledge of the team will not increase and the team innovation ability will decline. The model fits well the process of knowledge interaction in the actual interdisciplinary team and also illustrates the feasibility of system dynamics in the study of knowledge interaction. The model not only applies to the interdisciplinary teams, but also has some reference to all similar project teams. Because the process of knowledge interaction is a complicated process and its influencing factors cannot be considered thoroughly, some variables in the model are dealt with simply. The more complicated process of knowledge interaction in the model deserves further study and discussion in the future.

\section{Acknowledgement}

This research was supported by the National Natural Science Foundation of China (Grants nos. 71573064, 71273076, 71490724).

\section{References}

[1] A. P. Hare, Handbook of small group research (2nd ed.), New York: The Free Press, 1976. 
[2] G. W. Blackwell, Multidisciplinary team research // Chubin D E, et al., (Eds.), Interdisciplinary analysis and research, London: Lomond, 1986.

[3] M. J. Safoutin, D. L. Thurston, A communications-based technique for interdisciplinary design, IEEE Transactions on Engineering Management.40 (1993) 360-372.

[4] R. M. Grant, Prospering in dynamically-competitive environments: Organizational capability as knowledge integration, Organization Science. 7 (1996) 375-387.

[5] F. Toedtling, P. Lehner, A. Kaufmann, Do different types of innovation rely on specific kinds of knowledge interactions? Technovation. 29 (2009) 59-71.

[6] S. Pontis, A. Blandford, Understanding “influence:” An exploratory study of academics' processes of knowledge construction through iterative and interactive information seeking, Journal of the Association for Information Science and Technology, 66 (2015) 1576-1593.

[7] H. H. Wang, X. C. Jiang, F. J. Xie, Empirical study on the influential factors of inter-organization knowledge sharing under open innovation model, Science of science and management of S.\&T. 34 (2013) 83-90. (In Chinese)

[8] B. Z. Li, H. M. Dong, Research on the Influence Mechanism of Team Introspection on Team Knowledge Sharing Ability_—Role of Transactive Memory System and Regulation role of Social Capital, Science \& Technology progress and policy. 34 (2017) 120-126. (In Chinese)

[9] K. Sun, Y. T. Liu, X. T. Liu, The Influence of the R\&D Team Knowledge Heterogeneity on Knowledge Sharing, Information Science. 34 (2016) 59-64. (In Chinese)

[10]S. A. Zahra, G. George, Absorptive capacity: a review, reconceptualization and extension, Academy of Management Review. 27 (2002) 185-203. 